\title{
Image-Based Face Detection and Recognition
}

\author{
Gaurav Melkani ${ }^{1} \mid$ Dr. Sunil Maggu ${ }^{2}$ \\ ${ }^{1}$ IT (Information Technology), Maharaja Agrasen Institute of Technology, \\ ${ }^{2}$ Assistant Professor, IT (Information Technology), Maharaja AgrasenInstitute of Technology,
}

To Cite this Article

Gaurav Melkani and Dr. Sunil Maggu, "Image-Based Face Detection and Recognition", International Journal for Modern Trends in Science and Technology, 6(12): 465-470, 2020.

\section{Article Info}

Received on 16-November-2020, Revised on 09-December-2020, Accepted on 12-December-2020, Published on 20-December-2020.

\section{ABSTRACT}

Face recognition from image or video is a popular topic in biometrics research. Many public places usually have surveillance cameras for video capture and these cameras have their significant value for security purpose. It is widely acknowledgedthatthefacerecognitionhaveplayedanimportant role in surveillance system as it doesn't need the object's cooperation. The actual advantages of face based identification over other biometrics are uniqueness and acceptance. As human face is a dynamic object having high degree of variability in its appearance, that makes face detection a difficult problem in computer vision. In this field, accuracy and speed of identification is a mainissue.

The goal of this paper is to evaluate various face detection and recognition methods, provide complete solution for image based face detection and recognition with higher accuracy, better response rate as an initial step for video surveillance. Solution is proposed based on performed tests on various face rich databases in terms of subjects, pose, emotions, race andlight.

KEYWORDS: FaceDetection,FaceRecognition,Biometrics, Face Identification

\section{INTRODUCTION}

Over the last few decade lots of work is been done in face detection and recognition [14] as it's a best way for person identification [16] because it doesn't require human cooperation [15] so that it became a hot topic in biometrics. Since lots of methods are introduced for detection [6,7,8,12,13] and recognition $[8,9,10,11]$ which considered as a milestone. Although these methods are used several times for the same purpose separately for limited number of datasets in past but there is no workfoundwho provides overall performance evaluation of said methods altogether by testing them on tough datasets like $[1,2,3,4,5]$, details of datasets will be provided in sectionIV percentages determine the outcome of the match and the difference between good players and other players
(Donald, 2009).A tough tournament or competition challengesathletes' physical, psychological, and physiological positions. After intensive muscular exercise, structural muscle damage that is a major limiting factor for muscle activity, even for athletes who have not been injured, is observed (Ascensao et al, 2011).

Such exercise-induced muscle damage (EIMD)is characterized by decreased isometric muscle strength, change in joint motion, changes in muscle diameter, and muscle protein transfusions (Jakeman et al., 2009). CreatineKinase (CK) and LactateDehydrogenase(LDH)aretwoenzymesthat play a vital role in the metabolism of skeletal muscle,especiallyinseveremuscleactivities. These enzymes leave skeletal muscle and enter the bloodstream during damage. Therefore, these two 
enzymes are known as physiological indicators of muscle damage. The release of these two enzymes from the intramuscular medium toward the bloodstream indicates structural damage to muscle fibers (Brancaccio et al.,2008).

Exercise takes several days or a week, but microscopicandfinemuscledamageprobablydoes not cause immediate pain. Soreness may be delayed until 48-72 hours after the end of the exercise, as it may cause biochemical changes; therefore, muscle activity can alter the blood concentration of certain cellular enzyme such as CK and LDH. High plasma levels of these enzymes are commonly used as indicators of muscle tissue damage.

One of the main concerns of soccer coaches and players is the limited distance between activities and sports competitions for physiological recovery andrestoration of muscles to pre-exercise condition. Additionally, soccer players sometimes have to compete on consecutive days or even on a single day. In addition to theintensive activities, it imposes a lot of pressure on the musculoskeletal system of the athletes, which ultimately leads to a decline in their performance. Coaches, therefore, try to reduce the length of the recovery process in different ways and quickly prepare the athlete for the next competition. Among various recovery methods, immersion into water of different temperatures (cold, hot, subsequent cold and hot) is popular among athletes. Cold Water Immersion (CWI) is a popular method of cold therapy that is known as a potential effective anti-inflammatory treatment (Hamedinia et al., 2002).

Many methods have been used to reduce the undesirable effects of dangerous sports activities, although the degree of impact of these interventions is ambiguous. Most importantly, some of these methods may have a negative effect on short-term adaptations resulting from exercise activity. Therefore, in reviewing the recovery process, both aspects of restorationandadaptation should be considered. Therefore, the recovery periodshouldbeconsiderednotonlyasareturnto the initial state, but also as a new look at two stages of restoration and short-term adaptation of the training session.Immersion into cold water is one of the methods that have recently become popular in professional sports environments, although this recovering method is used whileits benefits and mechanism are still not scientifically well-known. Today, the use of water immersion among athletes has become prevalent in order to accelerate recovery after exercise (Gayiniet al., 2014).
Typically, the physiological changes occur in the bodyastheresultofcooling. Forexample, thebody temperature slowly returns to its normal level, so thattheheartandthecirculatorysystemreturnto thenormalstateslowly, andthedischargeofacidic products and materials derived from the metabolism ofmuscle cells rapidly occurs. The length and tension of the muscle are quickly returned to the original state, and the time of recovery after exercise is reduced and the internal fluid of the joints can easily be absorbed by tissues. This issue will be of particular importance in preventing damage to the joint and optimized performance.

Therefore, considering the expressed issues and factors as a problem, this study attempts to investigate the muscledamage caused by a soccerlike competition and the result of cold water immersion.

\section{METHODOLOGY}

AdaBoost [6] classifier is used with Haar [7] and Local Binary Pattern (LBP) [8] features whereas Support Vector Machine (SVM) [12] classifier is used with Histogram of Oriented Gradients (HOG) [13] features for face detectionevaluation. Haar-like [7] features are evaluated through the use of a new image representation that generates a large set of features and uses the boosting algorithm AdaBoost [6] to reduce degenerative tree of the boosted classifiers for robust and fast interferences only simple rectangular Haar- like [7] features are used that provides a number of benefits like sort of ad-hoc domain knowledge is implied as well as a speed increase over pixel based systems, suggestive to Haar [7] basis functions equivalent to intensity difference readings are quite easy to compute. Implementation of a system that used such features would provide a feature set that was far too large, hence the feature set must be only restricted to a small number of critical features which is achieved by boosting algorithm, Adaboost[6]. The original LBP [8] operator labels the pixels of an image by thresholding the 3-by-3 neighborhood of each pixel with the center pixel value and considering the result as a binary number. Each face image can be considered as a composition of micro-patterns which can be effectively detected by the LBP [8] operator. To consider the shape information of faces, they divided face images into $\mathrm{N}$ small non-overlapping 
regions TO, T1, .., TN. The LBP [8] histograms extracted from each sub-region are then concatenated into a single, spatially enhanced feature histogram definedas:

$H i, j=\Sigma x, y I(f l(x, y)=i) I((x, y) \in T j)$

where $i=0, \ldots, L-1 ; j=0, \ldots, N-1$. The extracted feature histogram describes the local texture and global shape of face images.

\begin{tabular}{|l|l|l|}
\hline 180 & 176 & 168 \\
\hline 179 & 175 & 170 \\
\hline 169 & 174 & 170 \\
\hline \multicolumn{3}{|c}{ Example }
\end{tabular}

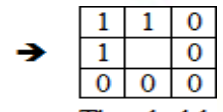

$(10000011)^{2}=13$ Pattern

Fig. 2 LBP calculation.

SVM [12] classifier is been used with HOG [13] features forfacedetection. HOG[13] greatly outperformswaveletsand degree of smoothing before calculating gradients damages, results emphasizes much of the available information is from sudden edges at fine scales that blurring this for reducing the sensitivity to spatial position is a mistake. Gradients should be calculated at the finest available scale in the current pyramid layer and strong local contrast normalization is essential for good results. Whereas SVM [12] are formulated to solve a classical two class problem which returns a binary value, the class of the object. To train our SVM [12] algorithm, we formulate the problem in a difference space that explicitly captures the dissimilarity between two facial images. The results summery of above methods are statedbelow.

Table 1: Face detection results summery

\begin{tabular}{|c|r|r|r|}
\hline \multirow{2}{*}{ Dataset } & \multicolumn{3}{|c|}{ Detection } \\
\cline { 2 - 4 } & \multicolumn{2}{|c|}{ Adaboost } & SVM \\
\cline { 2 - 4 } & Haar & LBP & HOG \\
\hline$[\mathbf{1}]$ & $99.31 \%$ & $95.22 \%$ & $92.68 \%$ \\
\hline$[\mathbf{2}]$ & $98.33 \%$ & $98.96 \%$ & $94.10 \%$ \\
\hline$[\mathbf{3}]$ & $98.31 \%$ & $69.83 \%$ & $87.89 \%$ \\
\hline$[\mathbf{4}]$ & $96.94 \%$ & $94.16 \%$ & $90.58 \%$ \\
\hline$[\mathbf{5}]$ & $90.65 \%$ & $88.31 \%$ & $89.19 \%$ \\
\hline Mean & $\begin{array}{c}\mathbf{9 6 . 7 0} \\
\text { \% }\end{array}$ & $\begin{array}{c}\mathbf{8 9 . 3 0} \\
\text { \% }\end{array}$ & $\begin{array}{c}\mathbf{9 0 . 8 8} \\
\%\end{array}$ \\
\hline
\end{tabular}
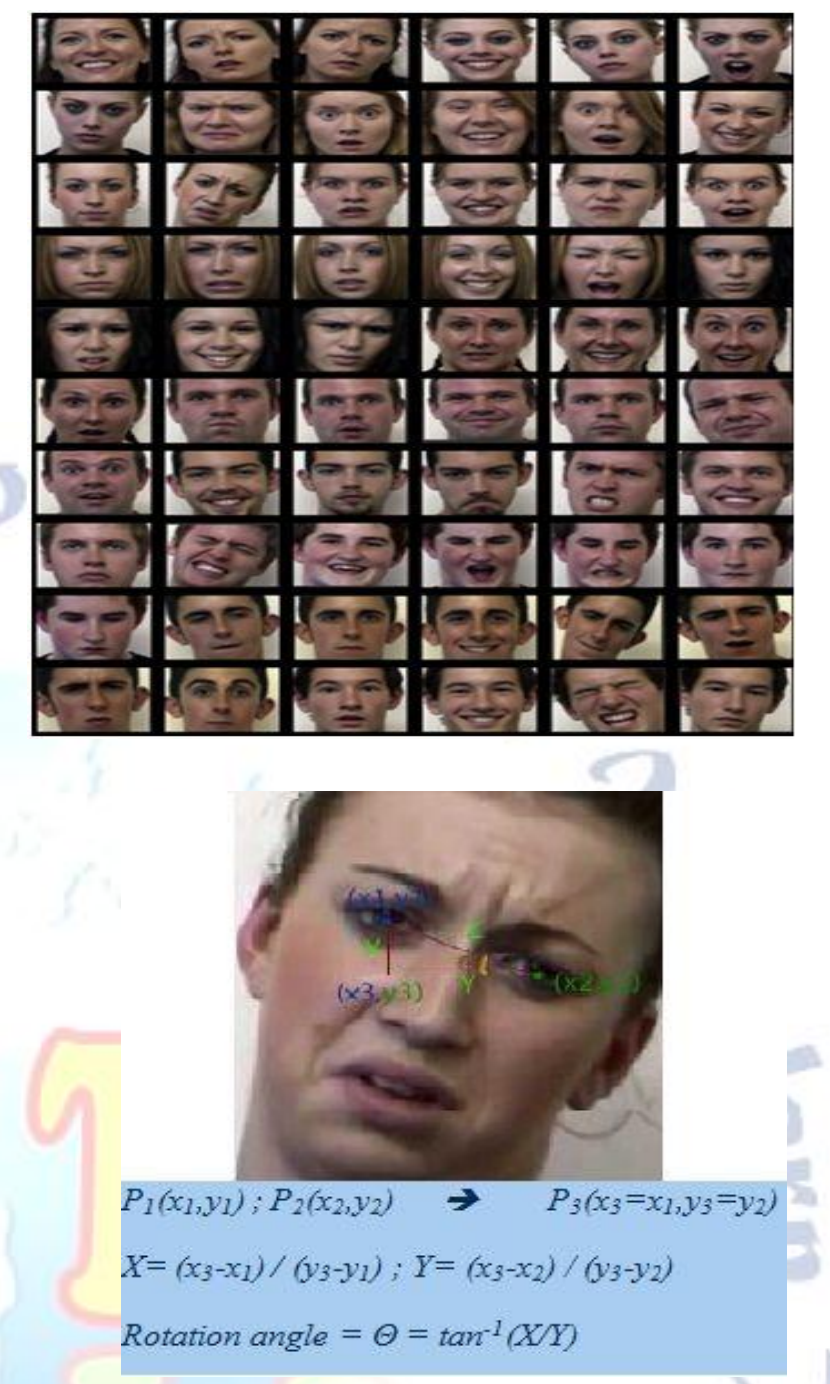

Moreover system is been tested on datasets $[1,2,3,4,5]$ and based on above demonstrated system, results are demonstrated below: To reduce pose variation and illumination in extracted faces two extra actions performed in pre-processing stage to improve recognition results: 1) Eyes detection isbeen used to remove head turn, tilt, slant and position of face, demonstrated in figure 4; 2) Histogram equalization is beenperformed.

\section{Facerecognition}

Eigenfaces [9] considered as 2-D face recognition problem, faces will be mostly upright and frontal. That's why 3-D information about the face is not required that reduces complexity by a significant bit. It convert the face images into a set of basis functions which essentially are the principal components of the face images seeks directions in which it is more efficient to represent the data. This is mainly useful for decrease the computational effort. Linear discriminant analysis is primarily used here to reduce the number of 
features to a more manageable number before recognition because face is represented by a large number of pixel values. Each of the new dimensions is a linear combination of pixel values, which form a template. The linear combinations obtained using Fisher's linear discriminant are called Fisherfaces [10]. LBP [8] is an order set of binary comparisons of pixel intensities between the center pixel and its eight surroundingpixels.

$$
L B P\left(x_{a}, y_{a}\right)={ }^{7} \sum_{n=0} s\left(i_{m}-i_{a}\right) 2^{n}
$$

Where $i_{a}$ corresponds to the value of the center pixel $\left(\mathrm{x}_{\mathrm{a}}, \mathrm{y}_{\mathrm{a}}\right), \mathrm{i}_{\mathrm{m}}$ to the value of eight surrounding pixels, function $\mathrm{f}(\mathrm{x})$ is definedas:

\section{1 if $x>=0$}

illumination, pose and expressions although Gabor [11] transform is not specially designed for face recognition. Its transformation formula is predefined instead of learned from the face training data. Moreover PCA[9] and LDA

classifier consider global features whereas LBP [8] and Gagor classifier consider local features, based on current facts experimental results are statedbelow.

Table 2: Face recognition results summery

\begin{tabular}{|c|c|c|c|c|}
\hline \multirow{2}{*}{ Dataset } & \multicolumn{4}{|c|}{ Recognition } \\
\cline { 2 - 5 } & PCA & LDA & LBP & Gabor \\
\hline$[\mathbf{1}]$ & $72.10 \%$ & $79.39 \%$ & $85.93 \%$ & $93.49 \%$ \\
\hline$[\mathbf{2}]$ & $69.87 \%$ & $76.61 \%$ & $80.47 \%$ & $89.76 \%$ \\
\hline$[3]$ & $70.95 \%$ & $78.34 \%$ & $84.14 \%$ & $92.68 \%$ \\
\hline$[4]$ & $74.79 \%$ & $81.93 \%$ & $86.45 \%$ & $96.91 \%$ \\
\hline$[5]$ & $68.04 \%$ & $73.21 \%$ & $77.69 \%$ & $88.93 \%$ \\
\hline Mean & $\mathbf{7 1 . 1 5 \%}$ & $\mathbf{7 7 . 9 0 \%}$ & $\mathbf{8 2 . 9 4 \%}$ & $\mathbf{9 2 . 3 5 \%}$ \\
\hline
\end{tabular}

\section{Dataset}

Five datasets been used for above experiments. In dataset [1], face collection with plain green background; no head scale and light variation but having minor changes in head turn, tilt, slant, position of face and considerable change in expressions.

In dataset [2], face collection with red curtain background, variation is caused by shadows as subject moves forward, having minor changes in head turn, tilt and slant; large head scale variation; some expression variation, translation in position of face and image lighting variation as subject moves forward, significant lighting changes occur on faces moment due to the artificial lighting arrangement. In dataset [3], face collection with complex background; large head scale variation; minor variations in head turn, tilt, slant and expression; some translation in face position and significant light variation because of object moment in artificial light. In dataset [4], face collection with plain background; small head scale variation; considerable variation in head turn, tilt, slant and major variation in expression; minor translation in face position and light variation. In dataset [5], face collection with constant background having minor head scale variation and light variation; huge variation in turn, tilt, slant, expressionand

\section{Conclusion}

In current work we developed the system to evaluate thefacedetectionandrecognitionmethodswhichare considered to be a bench mark. Some methods performed consistently over different datasets whereas other methods behave very randomly however based on average experimental results performance is evaluated, five datasets been used for this purpose. Face detection and recognition method's result summery is provided in table 1 and table 2 respectively whereas datasets summery is provided in table 3 . In current system Haar-like [7] features reported relatively well but it has much false detection than LBP [8] which could be consider being a future work in surveillance to reduce false detection in Haar-like [7] features and for the recognition partgabor

[11] is reported well as it's qualities overcomes datasets complexity.

Table 3: Face database summery

\begin{tabular}{|c|c|c|c|c|c|}
\hline $\begin{array}{c}\text { Data } \\
\text { Set }\end{array}$ & Sub-Division & $\begin{array}{c}\text { Image } \\
\text { S }\end{array}$ & $\begin{array}{c}\text { Resoluti } \\
\text { on }\end{array}$ & $\begin{array}{c}\text { Individua } \\
\text { ls }\end{array}$ & $\begin{array}{c}\text { Image/Individ } \\
\text { ual }\end{array}$ \\
\hline \multirow{4}{*}{ A } & Face 94 & 3078 & $180 * 200$ & 153 & $\sim 20$ \\
\cline { 2 - 6 } & Face 95 & 1440 & $180 * 200$ & 72 & 20 \\
\cline { 2 - 6 } & Face 96 & 3016 & $196 * 196$ & 152 & $\sim 20$ \\
\cline { 2 - 6 } & Grimace & 360 & $180 * 200$ & 18 & 20 \\
\hline \multirow{2}{*}{ B } & $\begin{array}{c}\text { Pain } \\
\text { Expressions }\end{array}$ & 599 & $720 * 576$ & 23 & 26 \\
\hline
\end{tabular}

A: Face Recognition Data, University of Essex

B: Psychological Image Collection at Stirling (PICS)

Acknowledgment

The work is being mainly done at Laboratory of Intelligent Recognition and Image Processing, Beijing University of Aeronautics and Astronautics. We would like to thank Dr Zhaoxiang Zhang for his guidance and support. $\mathrm{He}$ encouraged us to write this research paper. We are also grateful to our family for putting up with us. 


\section{REFERENCES}

[1] Face Recognition Data, University of Essex, UK, Face 94,

http://cswww.essex.ac.uk/mv/allfaces/faces94.html.

[2] Face Recognition Data, University of Essex, UK, Face 95,

http://cswww.essex.ac.uk/mv/allfaces/faces95.html.

[3] Face Recognition Data, University of Essex, UK, Face 96 ,

http://cswww.essex.ac.uk/mv/allfaces/faces96.html

[4] Face Recognition Data, University of Essex, UK, Grimace,

http://cswww.essex.ac.uk/mv/allfaces/grimace.html.

[5] Psychological Image Collection at Stirling (PICS), Pain Expressions, http://pics.psych.stir.ac.uk/2D_face_sets.htm.

[6] K.T.Talele,S.Kadam,A.Tikare,EfficientFaceDetection using Adaboost, "IJCA Proc on International Conferencein Computational Intelligence",2012.

[7] T. Mita, T. Kaneko, O. Hori, Joint Haar-like Features for Face Detection, "Proceedings of the Tenth IEEE International Conference on Computer Vision", 15505499/05 C2005IEEE.

[8] T.Ahonen,A.Hadid,M.Peitikainen,Facerecognitionwith local binary patterns. "In Proc. of European Conference of Computer Vision",2004.

[9] M. A. Turk and A.P. Pentland, Face recognition using eigenfaces, "Proceedings of the IEEE", 586-591,1991.

[10] J Lu, K. N. Plataniotis, A. N. Venetsanopoulos, Face recognition using LDA-based algorithms, "IEEE Neural Networks Transaction",2003.

[11] L. Wiskott, M. Fellous, N. Krger, and C. Malsburg, Face recognitionbyelasticbunchgraphmatching, "IEEETrans" , on PAMI, 19:775-779, 1997.

[12] I. Kukenys, B. McCane, Support Vector Machines for Human Face Detection, "Proceedings of the New Zealand ComputerScienceResearchStudentConference",2008.

[13] M. M. Abdelwahab, S. A. Aly, I. Yousry, Efficient WebBased Facial Recognition System Employing 2DHOG, arXiv: $1202.2449 \mathrm{v} 1[\mathrm{cs} . \mathrm{CV}]$.

[14] W. Zhao, R. chellappa, P. J. Phillips, Face recognition: A literature survey, "ACM Computing Surveys (CSUR)", December2003

[15] G. L. Marcialis, F. Roli, Chapter: Fusion of Face Recognition Algorithms for Video-Based Surveillance Systems, Department of Electrical and Electronic Engineering- Univ- ersity of Cagliari-Italy

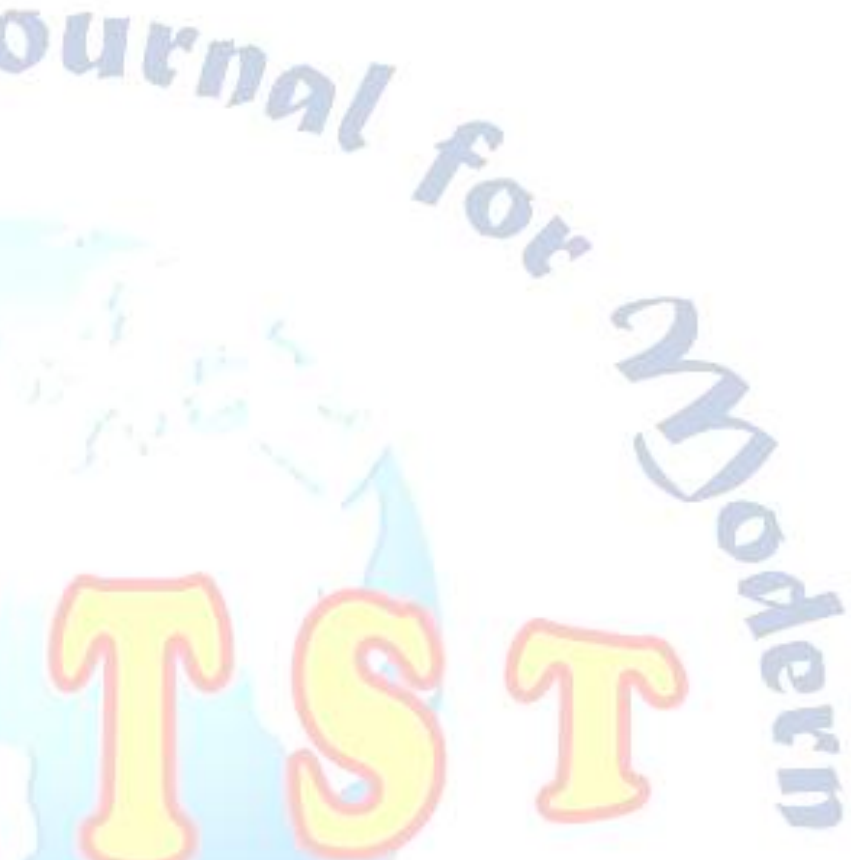

